\title{
Perfect mode locking of solid-state lasers by a double passive modulation
}

\author{
O. E. Martinez, ${ }^{a)}$ M. C. Marconi, ${ }^{\text {) }}$ and F. P. Dicdati \\ CEILAP, CITEFA-CONICET. Zufriategui y Varela- $1603 \mathrm{~V}$. Martelli Pcia. de Buenos Aires, Argentina
}

(Received 2 April 1982; accepted for publication 22 June 1982)

A new configuration, which incorporates a second passive modulation, has permitted complete selectivity in passive mode locking of solid-state lasers. A theoretical study is presented showing the existence of qualitatively different types of emission depending on the parameters of the system. Computed simulations are made to show the dependence of the emission on such parameters, and are used to determine their optimum range. Experimental results are presented confirming computed predictions.

PACS numbers: 42.60.By, 42.55.Rz

\section{INTRODUCTION}

A great amount of work has been done in the past decade in order to obtain reliable passive mode-locking of pulsed solid-state lasers. ${ }^{1-5}$ The main problem encountered in such systems stems from the statistical nature of the emission, as was pointed out in several theoretical studies. ${ }^{6-10}$ The statistical behavior is a result of the saturable absorner having to quench all but the most intense spike from the fluctuating initial noise pattern. The reliability of the system depends on its capability to select one of several pulses which initially have very similar intensities. With a numerical study, New" ' has shown the importance of gain saturation to improve the selectivity. However, deterministic emission may be obtained from pulsed systems with very few longitudinal modes pumped above threshold, such as $\mathrm{CO}_{2}$ lasers ${ }^{12}$ and $\mathrm{cw}$ passive mode-locked dye lasers. ${ }^{13}$ In this last case, the fast relaxation of the gain gurantees the complete selectivity without the appearance of relaxation oscillations. These relaxation oscillations inhibit passive mode-locking of cw solid-state lasers with saturable absorbers, unless they are stopped.

In a previous letter, ${ }^{14}$ a new configuration that leads to deterministic passive mode-locking of solid-state lasers was described. In this paper, a more detailed study of the system is presented, and the experimental results are given which confirm the conclusions obtained through the calculations.

The new configuration differs from the conventional passive mode-locking scheme in that a second passive modulator is inserted into the cavity. This extra modulator adds a loss proportional to the mean power in the cavity, with a relaxation time proportional to the round trip transit time. This loss prevents the system from $Q$-switching, and keeps the oscillation conditions just slightly above threshold. If the parameters are correctly adjusted, the smaller pulses in the initial noise pattern will be drawn below threshold by the losses introduced by the higher ones. The highest peak, which suffers the smallest absorption in the saturable absorber, will go growing while the other pulses finally disappear. In this way, the system may reach a stationary solution with only one pulse per round trip. The statistical behavior is hence replaced by a deterministic one, and in every laser

\footnotetext{
Present address: Bell Laboratories, Holmdel, New Jersey 07733 h) CONICET fellow.
}

shot, perfect mode-locking is achieved.

In Sec. II, we introduce the fundamental equation to study the evolution of the radiation. A qualitative discussion of the solutions is presented in Sec. III, and the different types of operation conditions are described. Numerical results are shown in Sec. IV, where the range of operation for the different parameters is determined. In Sec. V, the experimental setup is described, and the results are shown for different values of the parameters.

\section{THE BASIC EQUATIONS}

A typical cavity configuration is shown in Fig. 1. In some previous theoretical studies ${ }^{15.10}$ uniformly distributed amplification and absorption were assumed. This simplification gives a good quantitative approach if the variation of the radiation field after passing each element in the cavity is small. An equivalent approach ${ }^{6,16}$ is to evaluate the transmission of each component, and to expand the resulting exponentials to first order upon the assumption that radiation is only slightly modified after passing through each component. This is not the case for solid-state lasers in which the change after one passage through either the amplifier or SA is greater than 50 percent.

A more rigorous approach is to use a rate equation in which every component may change the radiation field to any degree, although the overall gain after each transit is still very small. This will be valid until $Q$-switching takes place and it is necessary to have good selectivity, because the selection of one pulse would take place before the bleaching of the dye.

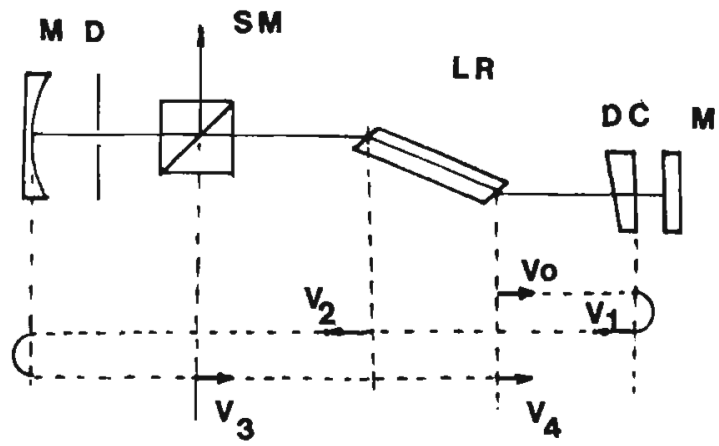

FIG. 1. Typical cavity configuration. $M: 100$ percent reflecting mirrors. $D$ mode selecting diaphragm. $S M$ : second modulation. $L R: N \mathrm{~N}$ :glass laser rod. $D C$ : dye cell contacted with the plane mirror. $v_{0}, v_{1}, v_{2}, v_{3}$, and $v_{4}$ are the different amplitudes of the electric field after passing each component in the cavity. 
For the saturable absorber, we will suppose that the relaxation time is much shorter than the pulse width (inertia less absorber) and use the expression given by Hercher. ${ }^{17}$

$$
\ln \left(I_{t} / I_{c}\right)+\left(I_{0}-I_{t}\right) / I_{s}=\alpha_{0},
$$

where $\alpha_{0}$ is the small-signal absorption coefficient, $I_{s}$ is the photon saturation intensity, $I_{c}$ is the input intensity, and $I_{l}$ is the corresponding output intensity. Demokan and Lindsay ${ }^{18}$ have shown that if a two-mirror cavity is used with a thin cell contacted with one mirror, the saturation intensity $I_{s}$ should be replaced by an equivalent saturation intensity $I_{s} / 2$, because of the superposition of the incoming pulse with its mirror reflection. Moreover, if $I$ is taken as the intensity in the laser medium, an additional factor $\rho$ should be included that accounts for the ratio between the beam area in the amplifying medium and the area in the saturable absorber.

If we define $v$ as the slowly varying envelope of the electric field, that is

$$
E(t) \alpha v(t) e^{-i w_{n} t},
$$

where $w_{0}$ is the central frequency of the radiation, and assuming that no phase distortion takes place after passing through the saturable absorber cell, Eq. (2.1) may be written as

$$
v_{1}=t_{0} v_{0} \exp \left|\left(\left|v_{0}\right|^{2}-\left|v_{1}\right|^{2}\right) / 2\right|,
$$

where $v_{1}$ is the transmitted amplitude (see Fig. 1), $v_{0}$ is the input amplitude, and $t_{0}=\exp \left(-\alpha_{0} / 2\right)$ is the small-signal amplitude transmission. In Eqs. (2.2) and (2.3), $v(t)$ is normalized so that

$$
I=|v|^{2} I_{s} .
$$

Equation (2.3) gives an implicit solution for $v_{1}$. As it is convenient to use explicit solutions, the following approximate expression will be used for $\left|v_{0}\right|^{2}-\left|v_{t}\right|^{2}$ :

$$
\left|v_{0}\right|^{2}-\left|v_{t}\right|^{2} \simeq f\left(\left|v_{0}\right|^{2}\right)=\frac{2 B\left|v_{0}\right|^{2}}{1+\left(2 B|v|^{2} / \alpha_{0}\right)}
$$

in which $1-2 B=t_{0}^{2}$. In Fig. 2, Eq. (2.5) is compared with (2.3)for $t_{0}^{2}=0.5$.

For the amplifying medium, the following assumptions are made: (a) The pulse energy is far from saturating the gain in one passage. The amplification is then linear, and the saturation is produced by the repeated transit of the pulse in the cavity. (b) Polarization of the radiation is linear in the active medium and in the $\mathrm{SA}$. (c) The radiation bandwidth is much smaller than the laser medium bandwidth; it is centered in a homogeneously broadened laser line.

With all these assumptions, following Hauss, ${ }^{16}$ the transmitted amplitude after one transit through the laser rod may be written as:

$$
v_{2}\left(t^{\prime}\right)=\exp \left[1 / 2 g_{0}\left(1-\frac{1}{w_{L}} \frac{d}{d t}+\frac{1}{w_{L}^{2}} \frac{d^{2}}{d t^{2}}\right)\right] v_{1}(t)
$$

where $t^{\prime}=t+v l$ is the time $t$ retarded at the output of the amplifier, $v$ is the group velocity of light, and $l$ is the length of the amplifier, $w_{L}$ is the laser bandwidth. Due to the assumption (a), $g_{0}$ will be a slowly varying parameter. The term $d / d t$ corresponds to the dispersion in the active medium, and will be neglected because its effect is to modify the cavity round trip time $\left(T_{\text {cav }}\right)$, as has been pointed out by Hauss, ${ }^{16}$ and we

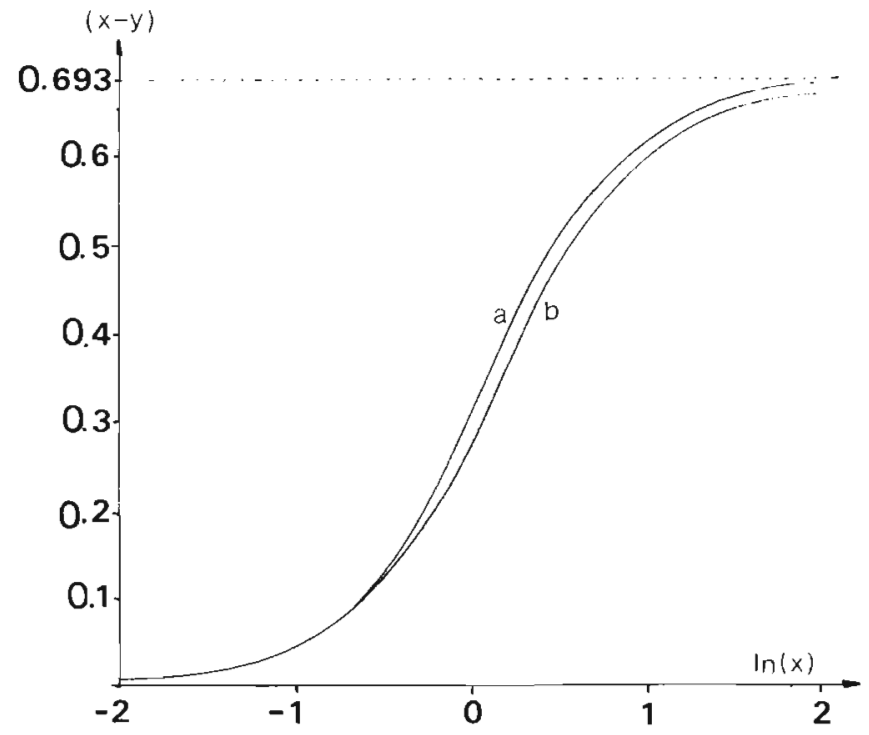

FIG. 2. Plot of the saturable absorber term. $x$ : input power. $y$ : output power. (a) Exact results given by Eq. (2.3). (b) Approximate result given by Eq. (2.5). For both curves, $t_{0}^{2}=0.5$.

are only interested in peak amplitude and pulse-width evolution.

All the losses except the one in the saturable absorber will be included in the second modulator loss. The transmission might be written as

$$
v_{3}=\exp (-\Gamma / 2) v_{2}
$$

where $\Gamma$ accounts for the linear losses and will be given by

$$
\Gamma=\Gamma_{0}+a V
$$

with

$$
V=\int_{0}^{T_{\text {cav }}}|v|^{2} d t
$$

That is, the losses are increasing linearly with the mean intensity in the cavity.

From now on, $t$ will be taken as a parameter that identifies one point between 0 and $T_{\text {cav }}$, and the time elapsed will be given by $t+T_{\text {cav }} \cdot k$, where $k$ counts the number of transmits in the cavity.

The amplitude after one round trip is then given by:

$$
\begin{aligned}
v(t, k+1)= & \phi\left\{\operatorname { e x p } \left[-\frac{\Gamma}{2}-\frac{\alpha_{0}}{2}+g_{0}\left(1+\frac{1}{w_{L}^{2}} \frac{\partial^{2}}{\partial t^{2}}\right)\right.\right. \\
& \left.\left.+\frac{B|v(t, k)|^{2}}{1+2 B / \alpha_{0}|v(t, k)|^{2}}\right]\right\} v(t, k),
\end{aligned}
$$

where $\phi$ is the ordering operator that always shifts the time derivatives to the left. This ordering is not essential if it is assumed that the radiation is only slightly modified after one round trip, and the exponential is developed to first order. In this case, Eq. (2.10) becomes

$$
\begin{aligned}
v(t, k+1)= & \mid 1+g_{0}\left(1+\frac{1}{w_{L}^{2}} \frac{\partial^{2}}{\partial t^{2}}\right) \\
& +\frac{B|v(t, k)|^{2}}{1+2 B / \alpha_{0}|v(t, k)|^{2}}-\frac{\Gamma}{2}-\frac{\alpha_{0}}{2} \mid v(t, k) .
\end{aligned}
$$


The pulse width $\tau$ may be estimated from the second derivative as follows:

$$
\left|\frac{V(t, k)}{\tau^{2}(k)}=-\frac{\partial^{2} v\left(t_{1} k\right)}{\partial t^{2}}\right|_{\text {peak }} .
$$

Taking the second derivative in Eq. (2.11), an equation for $\tau(k)$ is obtained. This equation will have a fourth derivative in $t$. If we write

$$
\left.\frac{\partial^{4} v}{\partial t^{4}}\right|_{\text {peak }}=\left.\frac{e v}{\tau^{4}}\right|_{\text {peak }},
$$

where $e$ depends on the pulse shape, the equation may be reduced to an equation containing only $v_{\text {peak }}$ and $\tau$. As an example, $e=4$ for a Gaussian pulse, and $e=5$ for a secanthyperbolic one. This last will be assumed for numerical computations.

Finally, the equations obtained are:

$$
\begin{aligned}
u_{n}(k+1)= & {\left[1+G(k)-4 a \tau_{1}(k) U(k)\right.} \\
& \left.+\frac{2 B u_{n}}{1+B^{\prime} u_{n}}-\frac{2 G_{0}(k)}{W_{L}^{2} \tau_{N}^{2}}\right] u_{n}, \\
\tau_{n}^{2}(k+1)= & \frac{4 G_{0}}{W_{L}^{2}}+\tau_{n}^{2}(k)\left(1-\frac{2 B u_{n}}{\left(1+B^{\prime} u_{n}\right)^{2}}\right),
\end{aligned}
$$

where $G_{0}=2 g_{0}, u_{n}$ is the normalized intensity of the $n$th fluctuation peak $\left(u=|v|^{2}\right)$, and $\tau_{n}$ is its pulse width. $B^{\prime}=2 B / \alpha_{0}$ and $G$ is the small-signal gain at the center of the line and will depend on the pumping rate $D$ and on the gain saturation as:

$$
G(k+1)=G(k)+D-C \tau_{1} U G_{0} .
$$

Also

$$
G_{0}(k+1)=G_{0}(k)+D-C \tau_{1} U G_{0},
$$

where in Eqs. $(2.14),(2.16)$, and (2.17):

$$
U(k)=\frac{1}{\tau_{1}} \sum_{n} \mu_{n} \tau_{n}=\sum_{n} \mu_{n},
$$

and all pulses are assumed to have the same width. This last assumption is quite reasonable because all pulses are rapidly broadened, lossing their initial differences. Only in the last stage of $Q$-switching, the pulses are subject to an intensitydependent compression, and we are looking for conditions in which only one pulse remains in that stage.

If the laser line were inhomogeneously broadened, the pulse would burn a hole in the line, and the effective bandwidth $w_{L}$ would change with saturation. This effect would, in any case, be favorable for the selection process and pulse compression, so the assumption of constant $w_{L}$ would be more restrictive than the real case for Nd:glass lasers.

\section{QUALITATIVE DISCUSSION OF SOLUTIONS}

From Eqs. (2.14) and (2.15), it may be seen that a stable quasi-stationary solution may exist in which only one pulse remains per transit. This would be:

$$
\tau^{2}=\frac{4 G_{0}}{w_{L}^{2}} \frac{\left(1+B^{\prime} u_{1}\right)^{2}}{2 B u_{1}}
$$

$$
G(k)-4 a \tau u+\frac{2 B u}{1+B^{\prime} u}-\frac{2 G_{0} u}{w_{L}^{2} \tau^{2}}=0 .
$$

If the selection of one pulse per transit is produced when $u<1$, the equations obtained are:

$$
\begin{aligned}
& G(k)-4 a \tau u+B u=0, \\
& \tau^{2}=\frac{2 G_{0}}{w_{L}^{2} B u},
\end{aligned}
$$

and the stable solution is

$$
u=\frac{G}{B}\left\{2 \gamma-1-2[\gamma(\gamma-1)]^{1 / 2}\right\},
$$

with

$$
\gamma=\frac{8 a^{2} G_{0}}{w_{L}^{2} B^{2} G} .
$$

This solution exists if

$$
\gamma>1
$$

otherwise, no stable solution is possible. The other solution of Eqs. (3.3) and (3.4), that gives higher peak power and shorter pulses, is not stable upon perturbations, as was shown by Hauss ${ }^{16}$ for dye lasers. Eq. (3.7) gives the limit for which the losses introduced by the electro-optic modulator are higher than the transmission growth in the saturable absorber. This type of operation will be called class " $a$." In this case, complete selection is found, and a quasi-stationary regime with only one pulse per transit is obtained.

If $\gamma$ is somewhat below unity, a complete selectivity may also be obtained, but only with additional $Q$-switching, because no stationary solution is possible. Such a range for $\gamma$ may be obtained assuming that two pulses of equal amplitude remain in one round trip, and finding the range for $\gamma$ that gives stationary solution. This solution will obviously not be stable, because any difference introdued between the two pulses will cause the bigger one to be amplified, and the smaller one to dissipate until it disappears.

Let $u_{1}=u_{2}$ be the intensity of the two pulses. Then from Eq. (3.3):

$$
G-8 a \tau u_{1}+B u_{n}=0
$$

which, in a similar way as Eq. (3.4), leads to:

$$
\gamma>1 / 4 \text {. }
$$

This last inequality and Eq. (3.7) give the range from which complete selectivity may be guaranteed and $Q$-switching may occur (depending on how far above second threshold the system is pumped). This type of operation will be called class " $b$." Out of this range, complete selectivity cannot be assured and the system will work in the conventional mode, but with a higher selectivity because the additional loss keeps the laser near threshold.

Another way of obtaining class " $b$ " operation is to give a maximum value to the losses added by the $\operatorname{EOM}\left(\Delta \Gamma_{\max }\right)$. This saturation of the second modulation must take place somewhat below its stationary value, but after completing the selection process. In real systems, such as described in Sec. $V$, this saturation always occurs before the bleaching of the dye, so that this type of operation is easier than adjusting 
only the value of $\gamma$. In this case, the maximum value for $\Delta \Gamma$ is given from (3.3) and (3.5):

$$
\Delta \Gamma_{\max }<G\left(2 \gamma p 2[\gamma(\gamma-1)]^{1 / 2}\right\} .
$$

As will be shown, the class " $b$ " operation condition is easy to be set, despite the limits required by Eqs. (3.9) or (3.10), because during the selection process, before the pulse compression takes place, the term $4 a$ in (3.3) may become bigger than the term $B$. Thus operating in class " $a$ " guarantees complete selectivity, but as the pulse grows towards the stationary solution, the pulse may be shortened one order of magnitude, lowering the value of $4 a \tau$ below the value of $B$, and shifting the system to class " $b$ " operation. Hence the best experimental setup would be to operate with the parameters near the limit of class " $a$."

In the numerical simulations in Sec. IV, the different types of operation are shown by varying $\Delta \Gamma_{\max }$. or the parameter $\gamma$ through changing the parameter $a$ and the pumping rate $D$ that enters into the determination of $G$ in Eq. (3.6).

\section{NUMERICAL RESULTS}

Numerical simulations were done with typical parameters for Nd:glass laser systems. The parameters were those measured for the laser used in the experimental setup described in the following section.

The pulse width will be measured in units of the inverse of the laser bandwidth $1 / w_{L}=0.3 \mathrm{ps}$. Hence the parameter $a$ is replaced by

$$
A=4 a / w_{L}
$$

and

$$
\begin{aligned}
& T=w_{L} \tau, \\
& S=C / w_{L}=\sigma I_{s} / w_{L},
\end{aligned}
$$

where $\sigma$ is the stimulated emission cross section and $I_{s}$ is the saturable absorber saturation photon flux, divided by the ratio between the area of the mode in the amplifying medium and the saturable absorber.

In all cases, the following values were used:

$$
B=0.25, \quad G_{0}=0.75 \text {, and } S=2 \times 10^{-6} \text {. }
$$

The initial radiation consists of $N$ pulses of peak intensity $u_{n}$. The distribution of the intensity of such peaks has been given by Demokan and Lindsay. ${ }^{19}$ In our simulation, we have decided to use the distribution

$$
f(U)=U \exp (-U),
$$

because it is easier to manipulate, and as the selection process is deterministic (not statistical), this approximation will not introduce any spurious results. When used for the conventional mode, as this distribution has a smaller tail than the one given in Ref. 19, the different pulses will tend to have similar intensities, and the selectivity of the system would appear slightly lower than in real systems.

The initial values taken in all cases were: $N=500$, $u_{0}=6 \times 10^{-6}$, and $T(k=0)=14$. These parameters depend slightly on the pumping rate, but since they will not lead to different results, the same values for all calculations were used.

In Fig. 3, a set of numerical simulations is shown using
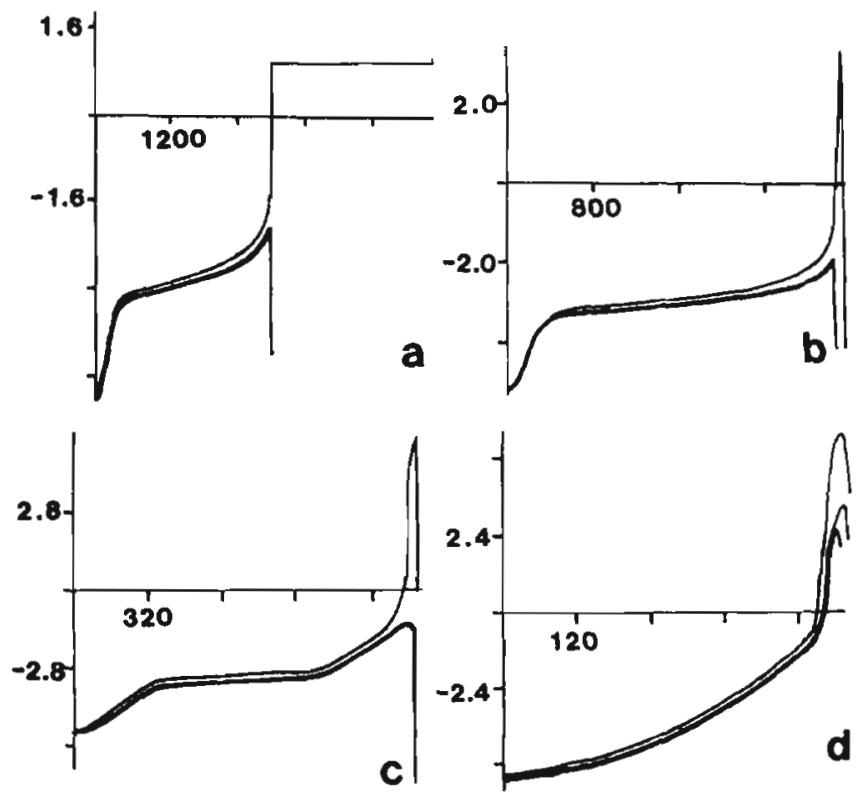

FIG. 3. Numerical simulation on varying $\Delta \Gamma_{\max }$. For the three highest pulses, $\ln (u)$ vs $k$ is plotted. The parameter values are: $B=0.25, G=0.75$, $S=2 \times 10^{-6}, N=500, u_{1}(k=0)=6 \times 10^{-6}, A=0.01, D=5 \times 10^{-3},(a)$ $\Delta \Gamma_{\max }=0.8$, (b) $\Delta \Gamma_{\max }=0.5$, (c) $\Delta \Gamma_{\max }=0.05$, (d) $\Delta \Gamma_{\max }$.

$D=5 \times 10^{-5}$ and $A=0.01$, for different values of $\Delta \Gamma_{\max }$ (the saturation value for $\Delta \Gamma$ ). In all cases, the lograrithm of the intensity of the three highest pulses are plotted versus the number of transits in the cavity $(k)$. For $\Delta \Gamma_{\max }=0.8$, class " $a$ " operation is found, and only one pulse is left that reaches a quasi-stationary solution. For smaller values of $\Delta \Gamma_{\max }$, complete selectivity is achieved, and the system simulta-

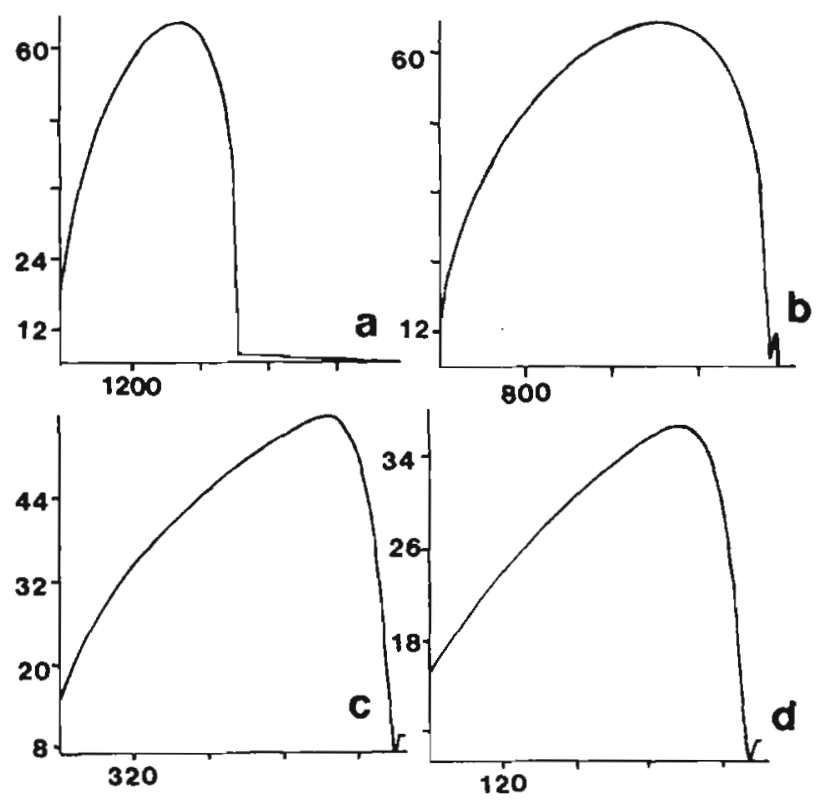

FIG. 4. Numerical simulation on varying $\Delta \Gamma_{\max }$. Pulse width evolution corresponding to Fig. 3. In all cases, the pulse width in units of the inverse of the laser bandwidth $1 / w_{L}$ vs $k$ is plotted. 
neously $Q$-switches ( $b$ and $c$ in Fig. 3), this corresponds to operation in class " $b$." If $\Delta \Gamma_{\max }$ is lowered even more, the system works in the conventional mode and the selectivity decreases [Fig. 3(d)]. All the curves were obtained with the same set of initial random pulses.

In Fig. 4, the corresponding evolution of the pulse width is shown. In all cases, the pulse is broadened during the first stage and at a peak power value of $u=0.1$, a fast pulse compression takes place until the intensity grows to about $u=10$; then a new broadening occurs because the dye is completely bleached, and it is no longer effective for shortening the pulse. Hence the final pulse width depends on the peak power, and it is shown that the pulse compression is enough to compensate for all the initial pulse broadening. The final pulse width does not depend on the type of operation, except for the fact that in class " $a$," the smaller intensity leads to a shorter pulse [Fig. 4(a)]. In this simulation, nonlinear effects in the laser medium were not taken into account. They have been discussed extensively in Refs. (20) and $(21)$, where it is shown that higher peak powers lead to wider pulses from dispersion in the nonlinear index of refraction. Anyway, we have shown numerically that even if those effects are neglected, the shortest pulse are the first ones in the train.

In Fig. 5, another set of simulations is shown, where $\Delta \Gamma_{\max }$ was kept constant at $\Delta \Gamma_{\max }=0.13$, and the pumping rate was changed. It is shown that as this parameter changes, one may obtain different types of operation. For small pumping rates, $D=10^{-5}$ [Fig. 5(c)], the net gain $G$ in Eq. (3.6) is kept low, and $\gamma$ grows enough to work in class " $a$." Higher values of $D$ give higher values of $G$ and the system works in class " $b$ " [Fig. 5(b)] and the conventional mode $(d)$. It may be concluded that there is an optimal range of $D$ if
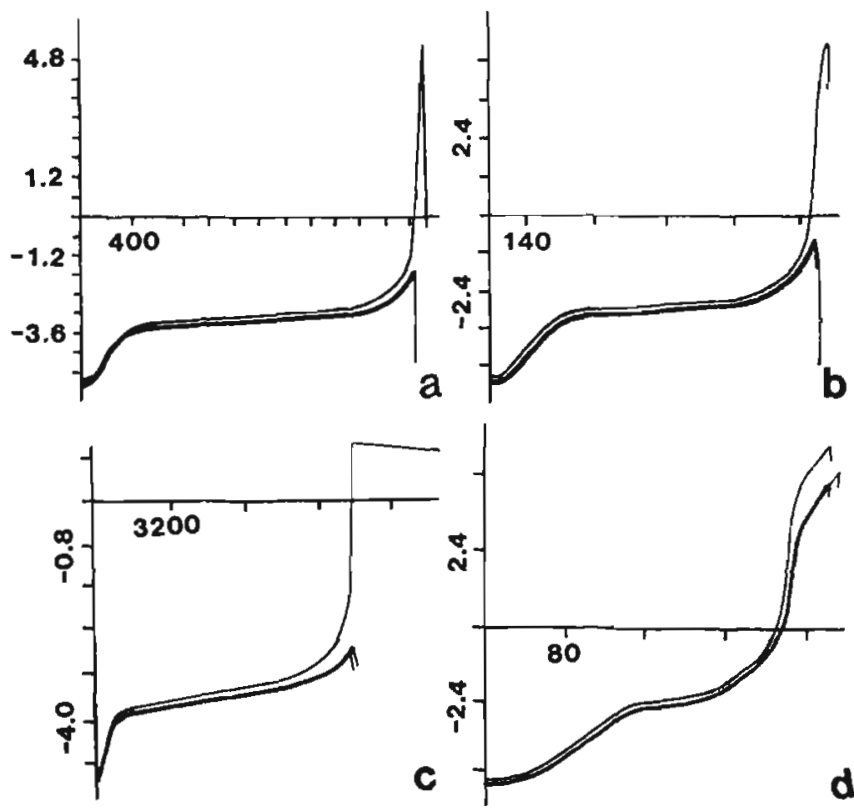

FIG. 5. Numerical simulations on varying $D$. For the three highest pulses, $\ln (u)$ vs $k$ are plotted. $B=0.25, G_{0}=0.75, S=2 \times 10^{-6}, N=500$, $u_{1}(k=0)=6 \times 10^{-65}, A=0.01, \Delta \Gamma_{\max }=0.13$, (a) $D=5 \times 10^{-, 5}$ (b) $D=10^{-4}$, (c) $D=10^{-5}$, (d) $D=5 \times 10^{-4}$.

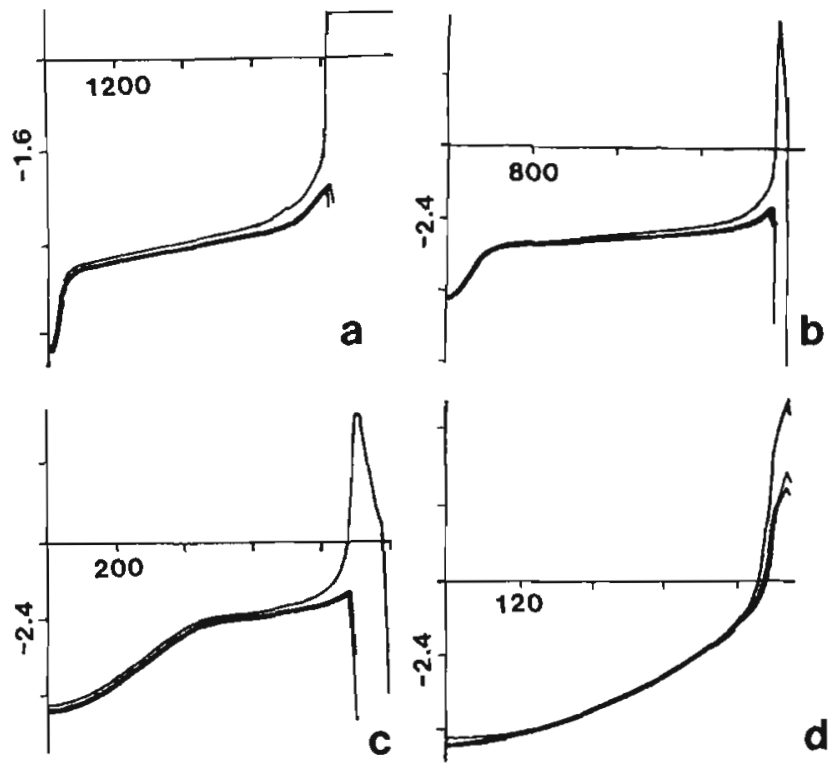

FIG. 6. Numerical results from varying $A$. $\ln (u)$ vs $k$ for the three highest pulses is plotted. $B=0.25, G_{0}=0.75, S=2 \times 10^{-6}, \quad N=500$, $\left.u_{1} \mid k=0\right)=6 \times 10^{-6}, \Delta \Gamma_{\max }=0.3, D=5 \times 10^{-5}$, (a) $A=2 \times 10^{-2}$, (b) $A=10^{-2}$, (c) $A=10^{-3}$, (d) $A=0$.

class " $b$ " operation is desired, but since the range required for high selectivity is less critical than in the conventional mode, it is no longer necessary to pump so close to the first threshold.

Numerical simulations of the evolution of the pulse width showed similar results to those of Fig. 4. The final pulse width depends strongly on the peak power, no matter how important the initial pulse broadening is.

In Eq. (3.6), it is easily seen that the parameter $a$, and hence $A$, also determines the type of operation. In Fig. 6, the set of simulations show that, operating in class " $b$," there is a very wide range for $A$ for which complete selectivity is achieved. In fact, either for $A=10^{-2}$ or $A=10^{-3}$, this condition is fulfilled. The simulation shows that operation in class " $b$ " does not require a critical adjustment of the parameters. $A, D$, and $\Delta \Gamma_{\max }$ have a range of one order of magnitude in which the emission does not change. As has been already pointed out, the optimum experimental setup would be to make the system work in class " $a$," during the selectivity process in which the pulse is broadened to about $T=50$ or more, and then to choose the parameter $A$ to make the system to shift to class " $b$," after the pulse shortening to about $T=10$ takes place.

\section{EXPERIMENTAL RESULTS}

The experimental setup used is similar to that described in Ref. (14), and is shown in Fig. 7. The filter $f$ is used to prevent spurious light from reaching the photodiode. The mirror $m 3$ and the polarizer $p 12$ are used to adjust the parameter $A$. The mirrors $m 1$ and $m 2$ have 100 percent reflectivity, and the output is obtained from the losses in the polarizer $p 11$ after passing twice through the electro-optic 


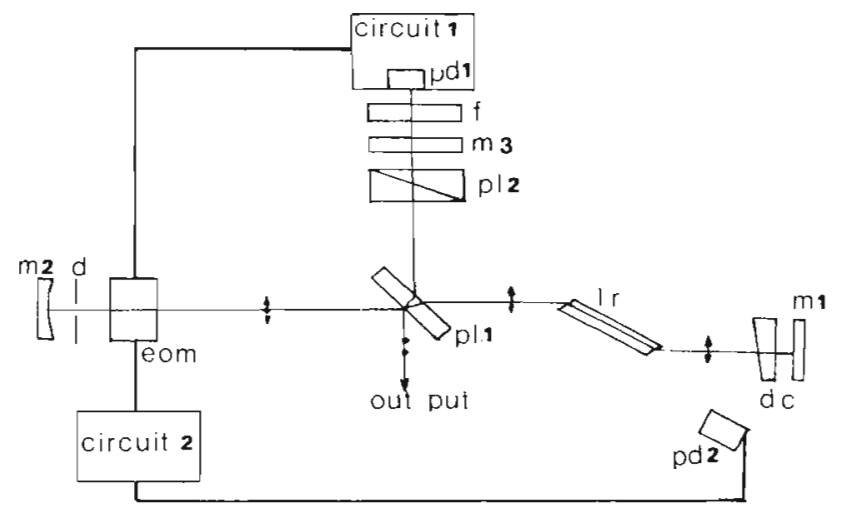

FIG. 7. Schematic of the experimental setup. /r: Nd:glass Br-Br laser rod. $d c$ : dye cell $p d$ 1,2: photodiodes. $m 1,2,3$ : dielectric mirrors. $d$ : mode selecting diaphragm $p l 1,2$ : polarizers. $f: 1.06-\mu$ filter. eom: electro-optic modulator.

modulator (EOM). The dye cell is $0.3-\mathrm{mm}$ wide and is contacted with the plane mirror. Circuit 2 is used to adjust the pumping rate $D$ in a similar way, as described in Ref. 22 . Circuit 1 is shown in Fig. 8. The photocurrent $i_{p}$ introduced by the photodiode yields an additional voltage drop:

$$
V=i_{p} R_{1}
$$

if the time constants $R_{1} C_{M}$ and the rise time of the photodiode are of the order of $T_{\text {cav }}$. This condition is satisfied with $R_{1}=560 \Omega$ and $c_{M} \cong 20 \mathrm{pF}$. The rise time of the photodiode EG\&G, SGD-444 is 10 ns and the sensitivity is $s=0.3 \mathrm{~A} / \mathrm{W}$ at $\lambda=1.06 \mu \mathrm{m}$. The parameter $a$ can be evaluated as

$$
a=\pi \tan \left(\phi_{0}\right) \frac{R_{1}}{V_{0}} S \frac{P_{S}}{T_{\text {cav }}} \delta,
$$

where

$$
\phi_{0}=\frac{V_{0}}{2 V_{\lambda / 4}} .
$$

$V_{0}$ is the voltage drop in the EOM at threshold, $V_{\lambda / 4}$ is the quarter-wave voltage of the EOM, $P_{s}$ is the saturation power of the SA, and $\delta$ is the fraction of the radiation power density in the cavity that reaches the photodiode. The Eq. (5.2) is obtained by evaluating the change in the losses introduced by an additional voltage drop in the EOM and taking into account the relation between $i_{p}$ in Eq. (5.1) and the power inside the cavity.

\section{CIRCUIT 1}

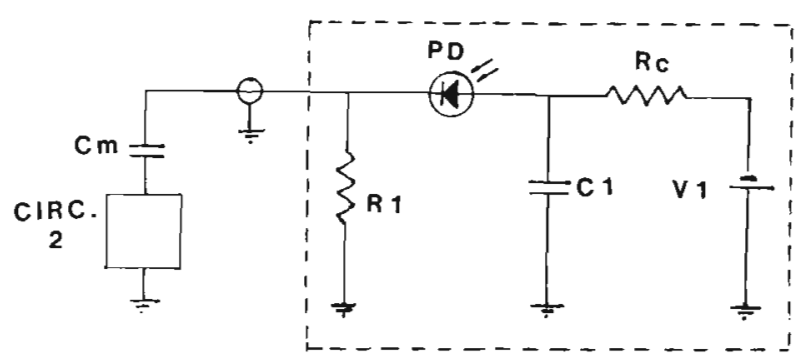

FIG. 8. Second modulation circuit. Corresponding to circuit 1 of Fig. 7. $R_{1}=560 \Omega . C_{M}:$ EOM's capacitance $\simeq 20$ pF. PD: EG\&G SGD-444 photodiode $C_{1}$ is a big capacitor which acts as a source charged by $V_{1}$ through $R_{c}$

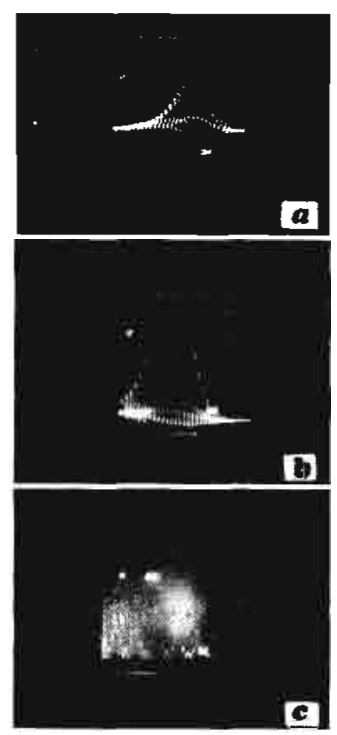

FIG. 9. Oscillagrams of typical pulse trains. (a) Conventional mode. $10 \mathrm{~ns} /$ div with reduced scan. (b) Class " $b$ " operation. $10 \mathrm{~ns} /$ div with reduced scan (c) Detail of the detector's oscillation. 2 ns/div with reduced scan. All oscillograms were obtained with a Textronix 7834 storage oscilloscope.

The pumping rate was fixed at $D=8 \times 10^{-5}$. It was evaluated taken into account the changes of the gain in the amplifying medium and the changes in the losses introduced by the EOM. With a typical value of $\tan \phi_{0}=1.5$, a value of $A=0.01$ is obtained if $\delta$ is fixed at $\delta=5 \times 10^{-4}$.

The maximum value for the additional voltage drop $V_{\max }$ is determined by $V_{1}$, as long as the photocurrent does not saturate. This is the case for the photodiode used because $i_{p}$ maximum is $1 \mathrm{~A}$ which would correspond to $560 \mathrm{~V}$, and much smaller values for $V_{\mathrm{I}}$ were used. For $V_{\mathrm{l}}=100 \mathrm{~V}$, $\Delta \Gamma_{\max }=0.15$ is obtained.

In Fig. 9, oscillograms of typical pulse trains are shown. Fig. 9(a) was obtained with the system working in the conventional mode, (i.e., $\delta=0$ ), several pulses per transit are observed. Fig. 9(b) shows the emission when $\delta=8 \times 10^{-4}$ and $V_{1}=100 \mathrm{~V}$. In Fig. $9(\mathrm{c})$, a similar pulse train is expanded

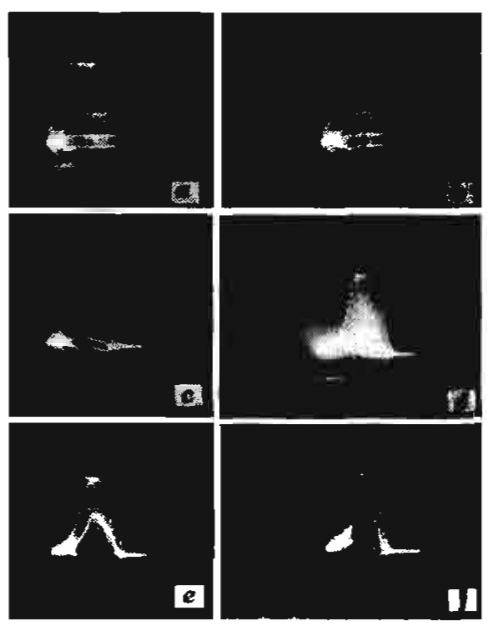

FIG. 10. Oscillograms of the pulses trains obtained on varying $\delta$. (a) $\delta=7 \times 10^{-3}$, (b) $\delta=2 \times 10^{-3}$, (c) $\delta=4 \times 10^{-4}$, (d) $\delta=1.4 \times 10^{-4}$, (e) $\delta=10^{-4},(\mathrm{f}) \delta=0$. 

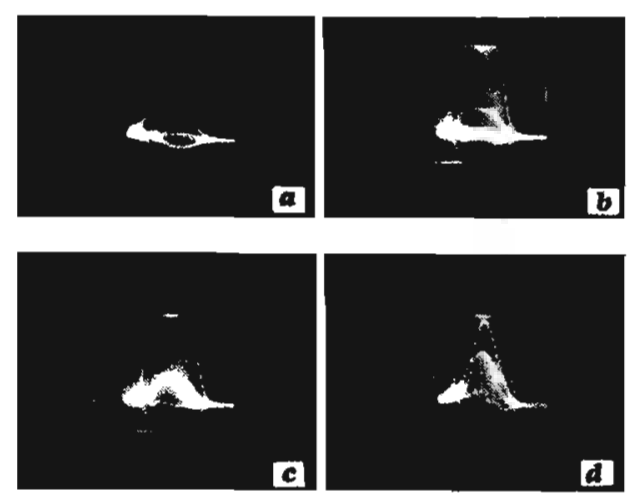

FIG. 11. Experimental results from varying $\Delta V_{\max }$. (a) $\Delta V_{\max }=200 \mathrm{~V}$, (b) $\Delta V_{\max }=100 \mathrm{~V}$, (c) $\Delta V_{\max }=50 \mathrm{~V}$, (d) $\Delta V_{\max }=25 \mathrm{~V}$.

showing the oscillation in the detection system. When perfect mode-locking is achieved, the oscillation appears neat, with two additional positive peaks and two negative peaks per round trip.

Different emission diagrams were obtained by changing parameters $\delta$ and $\Delta \Gamma_{\max }$. Figure 10 shows the pulse trains obtained for different values of $\delta$, which correspond to $A=0.14, A=0.04, A=2.8 \times 10^{-3}, A=2 \times 10^{-3}$, and $A=0$. These results show that for a wide range for $A$ between 0.14 and $8 \times 10^{-3}$, perfect mode-locking is achieved. As $\Delta \Gamma_{\max }$ is smaller than the value used in the numerical simulations of Fig. 5, the system does not operate in class " $a$," and the range for $A$ is slightly shifted towards higher values.

In Fig. 11, a similar set is shown changing $V_{1}$ from 200 $\mathrm{V}$ to $25 \mathrm{~V}$; that is, changing $\Delta \Gamma_{\max }$ from 0.3 to 0.04 . $A=5 \times 10^{-3}$ was kept for all the shots. Again, the range for $V_{1}$ is quite similar to that predicted in Fig. 4. Higher values are required because of the smaller value of $A$.

Operation in class " $a$ " was shown in Ref. 14 where higher voltage drops in the EOM and smaller pumping rates were used. $V_{1}=400 \mathrm{~V}$ and $D=2 \times 10^{-5}$ are typical values required for class " $a$ " operation. In this case, peak powers
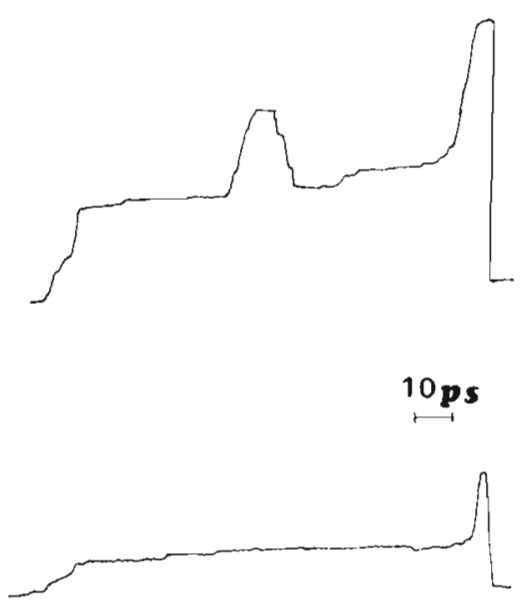

FIG. 12. Densitometer traces. First trace corresponds to the conventional mode with more than one pulse in the cell. Second trace corresponds to a pulse train obtained in class " $b$ " operation. more than three orders of magnitude smaller and very long pulse trains are obtained.

Pulse width measurements were made by two photon fluorescence using the scheme described in Ref. 23. In this scheme, the pulses are simply reflected upon themselves in a mirror immersed in a cell containing a solution of Rhodamine $6 \mathrm{G}$ dye in ethanol. In Fig. 12, two densitometer traces are shown. The first one shows a typical trace when the system operates in the conventional mode. As expected, more than one pulse is shown in the cell. The second densitometry trace corresponds to a pulse train obtained in class " $b$ " operation. A good contrast ratio of 3:1 is obtained, and no satellite pulses are observed. The mean pulse width over all the train is about 5 ps, which agrees quite well with the 3 ps predicted in the numerical simulations for the first pulses, before nonlinear effects start.

\section{CONCLUSIONS}

A novel technique for deterministic passive mode-locking of solid-state lasers has been studied in detail. A simplified rate-equation analysis has shown that several operation conditions are possible with qualitatively different emission patterns. They have been designed as: class " $a$," when a quasi-stationary solution is obtained with perfect mode-locking; class " $b$," when deterministic mode-locking is achieved, but with simultaneous $Q$-switching; and conventional mode, when the emission has a statistical nature.

Several parameters of the system, such as the pumping rate, the maximum losses introduced by the second modulation, and the parameter $a$, determine the operation conditions. The numerical simulations have shown that the ranges are quite large, so that it is not necessary to adjust the parameters critically, and quite simple experimental setups are possible. Experimental results have confirmed numerical predictions, and a high reliability has been achieved with the system proposed under conditions in which reliable conventional passive mode-locking would be impossible, such as, for example, a stability of about 10 percent in the energy delivered to the flashlamp.

Operation in class " $b$ " is desirable for pulsed laser systems because of the high peak power encountered, and for typical parameters, it is easier to obtain than operation in class " $a$," for which small pumping rates are required, i.e., pumping very near laser threshold.

As a last remark, it can be stated that operation in class " $a$ " would make it possible to mode-lock CW solid-state lasers. The relaxation oscillations that prevent passive modelocking in conventional systems ${ }^{13}$ can be eliminated by the second modulation if the losses introduced are higher than the growth of the transmittance in the dye from absorption saturation, which is precisely the condition for operation in class " $a$."

\section{ACKNOWLEDGMENTS}

The authors wish to thank Mr. Luis A. Spinelli, who as the Senior Engineer very easily got involved in the electronic circuitry design, and also for the very useful suggestions 
made during the discussion of the experiment. We are also indebted to all the members of the CEILAP.

'H. W. Mocher and R. S. Collins, Appl. Phys. Lett. 7, 270 (1965).

${ }^{2}$ A. J. DeMaria, D. A. Stetser, and H. Heynau, Appl. Phys. Lett. 8, 174 (1966).

${ }^{3}$ G. R. Fleming, I. R. Harrowfield, A. E. W. Knight, J. M. Morris, R. J. Robbins, and G. W. Robinson, Opt. Commun. 20, 36 (1977).

${ }^{4}$ W. Koechner, Solid State Laser Engineering, Springer Series in Optical Sciences (Springer-Verlag, New York, 1976), Vol. 1, p. 461.

${ }^{5}$ D. J. Bradley, in Ultrashort Light Pulses, edited by S. L. Shapiro (SpringerVerlag, New York, 1977), Vol. 18, p. 36.
'P. G. Kryulov and V. S. Letokhov, IEEE J. Quantum Electron. QE-8, 766 (1972).

'B. Ya. Zel'dovich and T. I. Kuznetsova, Sov. Phys. Uspekhi. 15, 25 (1972).

${ }^{k}$ J. A. Fleck, Jr., Phys. Rev. B 1, 84 (1970).

${ }^{\circ}$ M. S. Demokan and P. A. Lindsay, Int. J. Electron. 41, 421 (1976).

${ }^{10}$ G. H. C. New, Proc. IEEE, 67 (3), 380 (1979).

"G. H. C. New, IEEE J. Quantum Electron. QE-14, 642 (1978).

${ }^{12}$ C. P. Ausschnitt, IEEE J. Quantum Electron QE-13, 321 (1977).

${ }^{13}$ H. A. Hauss, IEEE J. Quantum Electron. QE-12, 169 (1976).

${ }^{14}$ O. E. Martinez and L. A. Spinelli, Appl. Phys. Lett. 39, 875 (1981).

${ }^{15}$ G. H. C. New, IEEE J. Quantum Electron. QE-10, 115 (1974).

${ }^{16}$ H. A. Hauss, J. Appl. Phys. 46, $3049\{1975\}$.

${ }^{17}$ M. Hercher, Appl. Opt. 6, 947 (1967).

'M. S. Demokan and P. A. Lindsay, Int. J. Electron. 42, 313 (1977).

${ }^{14}$ N. S. Demokan and P. A. Lindsay, Int. J. Electron. 42, 417 (1977).

${ }^{21} \mathrm{M}$. A. Duguay, J. W. Hansen, and S. L. Shapiro, IEEE J. Quantum ElecIron. QE-6, 725 (1970).

${ }^{21} D$. J. Bradley and W. Sibbett, Opt. Commun. 8, 91 (1973).

${ }^{22}$ M. C. Marconi, O. E. Martinez, and F. P. Diodati, Appl. Phys. Lett. 37, $684(1980)$.

${ }^{23}$ S. A. Ggiordmaine, P. M. Rentzepis, S. L. Shappiro, and K. W. Wecht, Appl. Phys. Lett. 11, 216 (1967). 